\title{
Low-Reynolds-number flow through two-dimensional shunts
}

\author{
A. SET T H I ${ }^{1} \dagger$, A. J. MESTEL $\mathbf{L}^{2}$, \\ K. P A RKER R AND J. S I G GER $\mathbf{S}^{1}$ \\ ${ }^{1}$ Department of Bioengineering, Imperial College London, London, SW7 2AZ, UK \\ ${ }^{2}$ Department of Mathematics, Imperial College London, London, SW7 2AZ, UK \\ (Received ?; revised ?; accepted ?. - To be entered by editorial office)
}

Motivated by numerous biological and industrial applications relating to bypasses, mixing and leakage, we consider low-Reynolds-number flow through a shunt between two channels. An analytical solution for the streamfunction is found by matching biorthogonal expansions of Papkovich-Fadle eigenfunctions in rectangular subregions.

The general solution can be adapted to model a variety of interesting problems of flow through two-dimensional shunts by imposing different inlet and outlet flux distributions. We present several such flow profiles but the majority of results relate to the particular problem of a side-to-side anastomosis in the small intestine. We consider different flux fractions through the shunt with particular emphasis on the pressure and shear stress distributions, which are important factors in estimating health risks pertaining to this surgical procedure.

Key words: Papkovich-Fadle eigenfunctions; Biharmonic equation; Side-to-side anastomosis; Low-Reynolds-number flow

\section{Introduction}

The human body relies on shunts (also referred to as anastomoses or fistulas) to either regulate the pressure and flow in the system or to bypass sites of high resistance or damage. A side-to-side intestinal anastomosis, in particular, is the surgical procedure of incising two parallel segments of gut and suturing them together (Figure 1). It is used to bypass the redundant loop in case of disease (for example Crohn's disease) or to achieve long-term weight loss by restricting the uptake of metabolites. It is the preferred procedure in newborns when the gut is small, in older patients when the vessels are of different diameters, or when the gut is difficult to mobilise because of adhesions. It has similar, and sometimes higher, success rates as alternative procedures (Kiran et al. 2011; Resegotti et al. 2005), in particular when several shunts along the interface of the two vessels are introduced.

Although side-to-side anastomosis is a relatively common surgical procedure, there is still a debate in the surgical community regarding the function of the shunts (Joyce et al. 2002). Motivated by the need to understand the mechanisms better, we consider a mathematical model of the flow through a side-to-side anastomosis. The small intestine is a convoluted tube of about 6-7 m. in length and has average radius of about $1.25 \mathrm{~cm}$. The rheology of the contents depends on the distance from the stomach. It is semi-liquid as

$\dagger$ Email address for correspondence: asetchi@imperial.ac.uk 


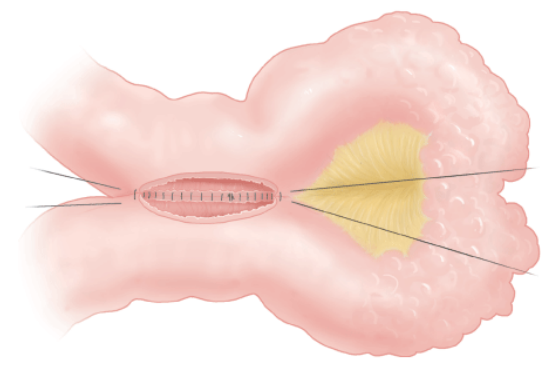

FIgURE 1. A diagram of a side-to-side anastomosis of the small intestine. Image from Souba et al. (2007).

it enters the small intestine from the stomach and the partially-digested material leaving it to go into the large intestine has a water content of around $95 \%$. We model the fluid as Newtonian, with the motivation that the results should identify the main dynamics if the consumed food is liquefied and we consider the flow a sufficient distance away from the stomach. Although there is a considerable debate about the typical Reynolds number of the flow, it is certainly small (Tripathi 2011). For water with typical residence time in the human small intestine, we estimate it as $10^{-3}$.

There exist several analytical models of Stokes flows in idealised geometries (Kim \& Karrila 2005; Pozrikidis 1992) but flow through shunts remains challenging near sharp edges and corners. Unbounded two-dimensional shear flow over a wall with a gap was studied analytically by Smith (1987). In addition, Ko \& Jeong (1994) found a solution in the same geometry but driven by a stagnation point flow. More recently, Crowdy \& Samson (2010) extended the solutions using complex variable techniques to flow past a wall with two gaps. Davis (1991) considered the three-dimensional flow over a planar wall with a circular orifice. The classical problem of channel flow divided by a semiinfinite barrier introduces additional boundaries, which complicates the solution. When the barrier halves the channel, the flow can be obtained by solving scalar Wiener-Hopf equations using an intricate factorisation (Buchwald \& Doran 1965; Foote \& Buchwald 1985). When it divides a channel asymmetrically, a solution can be obtained numerically using a Padé approximant technique for a matrix Wiener-Hopf system (Abrahams et al. 2008). In order to model flow through a shunt, we need to include a second barrier in a channel flow and allow for a general solution determined by the four fluxes at the geometry inlets/outlets. Although the Wiener-Hopf technique is commonly used for modelling Stokes flow in geometries with parallel boundaries, these complications would require a still more complex analysis than that presented by Abrahams et al. (2008) and we choose to use the method of Papkovich-Fadle eigenfunctions.

Papkovich-Fadle eigenfunctions were first used by Smith (1952) to obtain an exact solution for the normal displacement of a thin semi-infinite strip subjected to arbitrary displacements on the short edge but with the long edges clamped. Convergence of the series solutions was discussed by Joseph et al. (1982), Joseph \& Sturges (1978) and Joseph (1977). Since 1952 they have been used to model two-dimensional Stokes flow in rectangular domains with one inlet and one outlet. For example, Joseph \& Sturges (1975) studied the liquid circulation in a trench driven by unequal temperatures at the side walls. Then, Trogdon \& Joseph (1982) considered flow in an infinite channel over a rectangular slot. More recently, Phillips (1989) used the method of matched eigenfunction expansions to study Stokes flow in a channel with a contraction. A more recent application was by Driesen et al. (1998) and considered flow over partially covered cavities, and in particular etched holes. Our work is the natural extension to all these studies as it 


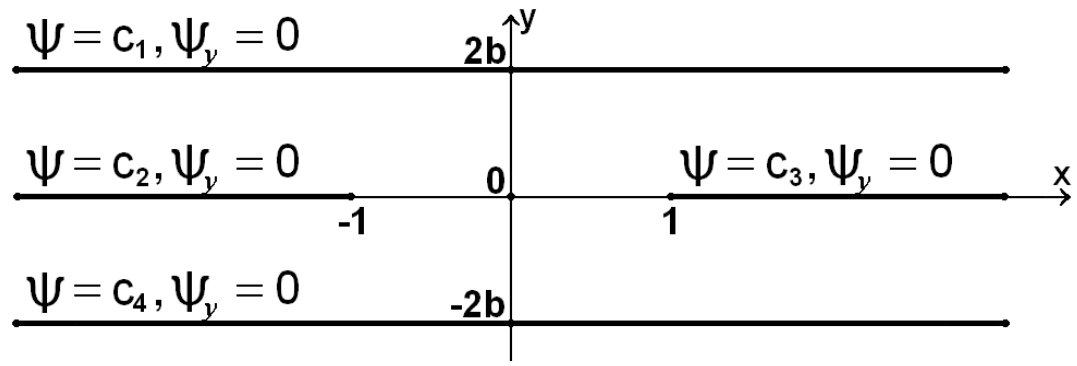

Figure 2. Geometry and boundary conditions associated with the streamfunction $\Psi(x, y)$.

The constants $c_{n}$ are determined by the Poiseuille flow fluxes at the inlets and outlets.

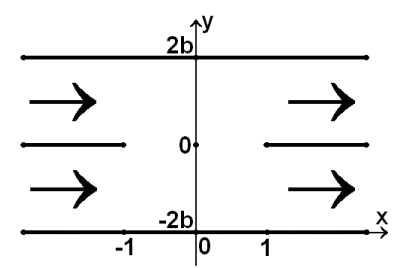

(a)

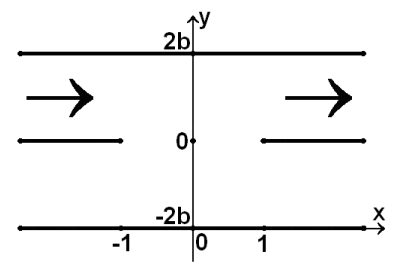

(b)

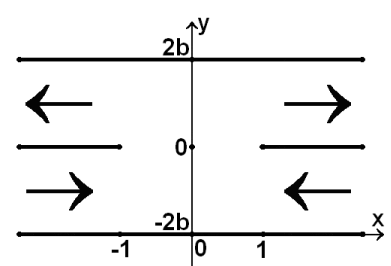

(c)

Figure 3. Schematic illustrating the three independent Stokes flow solutions that we consider in Sections 2.1, 2.2 and 2.3. The arrows represent Poiseuille flow of maximum velocity 1 at the extremities.

introduces a geometry with four channel ends rather than two, and thus the resulting matching analysis is more challenging.

We consider a two-dimensional geometry that consists of two channels joined by a slit. This is justified by the fact that the curvature of the vessels during a side-to-side anastomosis is negligible as the surgery is performed on parallel segments of the gut. A similar idealised geometry was used in previous work (Setchi et al. 2012) for highWormesely-number flow, where the flow was governed by Laplace's equation. For that problem, we found agreement between the results in this geometry and those in the midplane of two more realistic three-dimensional geometries: two cylinders joined by a point and two semi-cylinders joined by a rectangular shunt.

The simplified geometry allows an analytic solution of the biharmonic equation by splitting the domain into rectangular subregions and expressing the flow in each one using Papkovich-Fadle eigenfunction expansions. In reality it is peristalsis, i.e. the wavelike radial contraction and relaxation of the vessel walls, that drives the flow down the intestine but we only consider the effect of such a mechanism by prescribing the flow fluxes at the inlets and outlets.

\section{Mathematical model}

We consider Stokes flow in two parallel infinite channels of width $2 b$ linked by a side-toside anastomosis of length 2 (Figure 2). The flow is defined by a streamfunction $\Psi(x, y)$ such that $\mathbf{u}=\nabla \wedge(\Psi \mathbf{k})$, where $\mathbf{k}$ is a unit vector perpendicular to the flow plane. At low Reynolds numbers, the problem reduces to solving the biharmonic equation

$$
\nabla^{4} \Psi=0 \text {. }
$$

The flow is driven by suitable fluxes in and out of the four channel ends and satisfies the boundary conditions of vanishing $\partial \Psi / \partial x$ and $\partial \Psi / \partial y$ at the walls. The fluxes at the 
inlets and outlets are prescribed by the four constants $c_{1}, c_{2}, c_{3}$ and $c_{4}$ in Figure 2, which provide three constraints as a constant can be added to $\Psi$ without changing the solution. As in Setchi et al. (2012) we shall seek three linearly independent solutions for particular values of $c_{i}$ which we will then use as a basis for the general case. These three solutions are chosen so as to utilise the lines of symmetry of the geometry. Diagrams of these are shown in Figure 3, where the arrows represent Poiseuille flow profiles of flux 4b/3.

We seek separable solutions to the biharmonic equation. In doing so we find terms of the form $e_{1}^{n}(y) \exp \left(k_{n} x\right)$ and $e_{2}^{n}(y) \exp \left(K_{n} x\right)$, where

$$
\begin{aligned}
& e_{1}^{n}(y)=k_{n}\left(\cos k_{n} \sin \left(k_{n} y\right)-y \sin k_{n} \cos \left(k_{n} y\right)\right) \\
& e_{2}^{n}(y)=K_{n}\left(\sin K_{n} \cos \left(K_{n} y\right)-y \cos K_{n} \sin \left(K_{n} y\right)\right) .
\end{aligned}
$$

These are the odd and even Papkovich-Fadle eigenfunctions respectively. They have the properties that both they and their first derivatives are zero on $y=0$ and $y=1$ provided $k_{n}$ and $K_{n}$ are such that

$$
\begin{aligned}
\sin \left(2 k_{n}\right) & =2 k_{n} \\
\sin \left(2 K_{n}\right) & =-2 K_{n} .
\end{aligned}
$$

There are countably infinite sets of complex solutions to these equations. Note that if $\kappa$ is a solution to either of these equations, so are $-\kappa$ and their complex conjugates. We are interested in the roots in the first quadrant of the complex plane, which we number in ascending order of the real part. The Papkovich-Fadle eigenfunctions are useful when solving Stokes flows in rectangular geometries because they satisfy a biorthogonality property, which is utilised when matching flow expansions at an interface. This biorthogonality condition is written in terms of the Papkovich-Fadle eigenvectors

$$
\mathbf{e}_{1}^{n}(y)=\left[\begin{array}{c}
e_{1}^{n}(y) \\
e_{1}^{n \prime \prime}(y) / k_{n}^{2}
\end{array}\right] \text { and } \mathbf{e}_{2}^{n}(y)=\left[\begin{array}{c}
e_{2}^{n}(y) \\
e_{2}^{n \prime \prime}(y) / K_{n}^{2}
\end{array}\right]
$$

and respective adjoint eigenvectors

$$
\mathbf{f}_{1}^{n}(y)=\left[\begin{array}{c}
e_{1}^{n}(y)+2 \sin k_{n} \sin \left(k_{n} y\right) \\
e_{1}^{n}(y)
\end{array}\right] \quad \text { and } \quad \mathbf{f}_{2}^{n}(y)=\left[\begin{array}{c}
e_{2}^{n}(y)-2 \cos K_{n} \cos \left(K_{n} y\right) \\
e_{2}^{n}(y)
\end{array}\right](2,7)
$$

which are derived in detail by Smith (1952). Then the biorthogonality condition is

$$
\begin{aligned}
& \int_{-1}^{1} \mathbf{f}_{j}^{N}(y)^{T} \cdot\left[\begin{array}{cc}
0 & -1 \\
1 & 2
\end{array}\right] \cdot \mathbf{e}_{1}^{n}(y) d y=-4 \sin ^{4} k_{N} \delta_{j 1} \delta_{n N} \quad \text { for } j=1,2 ; \forall n, N \in Z^{+} \\
& \int_{-1}^{1} \mathbf{f}_{j}^{N}(y)^{T} \cdot\left[\begin{array}{cc}
0 & -1 \\
1 & 2
\end{array}\right] . \mathbf{e}_{2}^{n}(y) d y=-4 \sin ^{4} K_{N} \delta_{j 2} \delta_{n N} \quad \text { for } j=1,2 ; \forall n, N \in Z^{+}
\end{aligned}
$$

To ease notation in the rest of the paper we introduce the operator

$$
\langle\mathbf{u}(y), \mathbf{v}(y)\rangle=\int_{-1}^{1} \mathbf{u}^{T} \cdot\left[\begin{array}{cc}
0 & -1 \\
1 & 2
\end{array}\right] \cdot \mathbf{v} d y
$$

as well as the definitions

$$
\begin{gathered}
y_{1}=\frac{y}{b}-1, \quad y_{2}=-\frac{y}{b}-1, \quad y_{3}=\frac{y}{2 b}, \\
\tilde{f}(y)=\frac{y^{2}}{b}-\frac{y^{3}}{3 b^{2}} \quad \text { and } \quad f\left(y_{1}\right)=\frac{2 b}{3}+b y_{1}-\frac{b y_{1}^{3}}{3}
\end{gathered}
$$



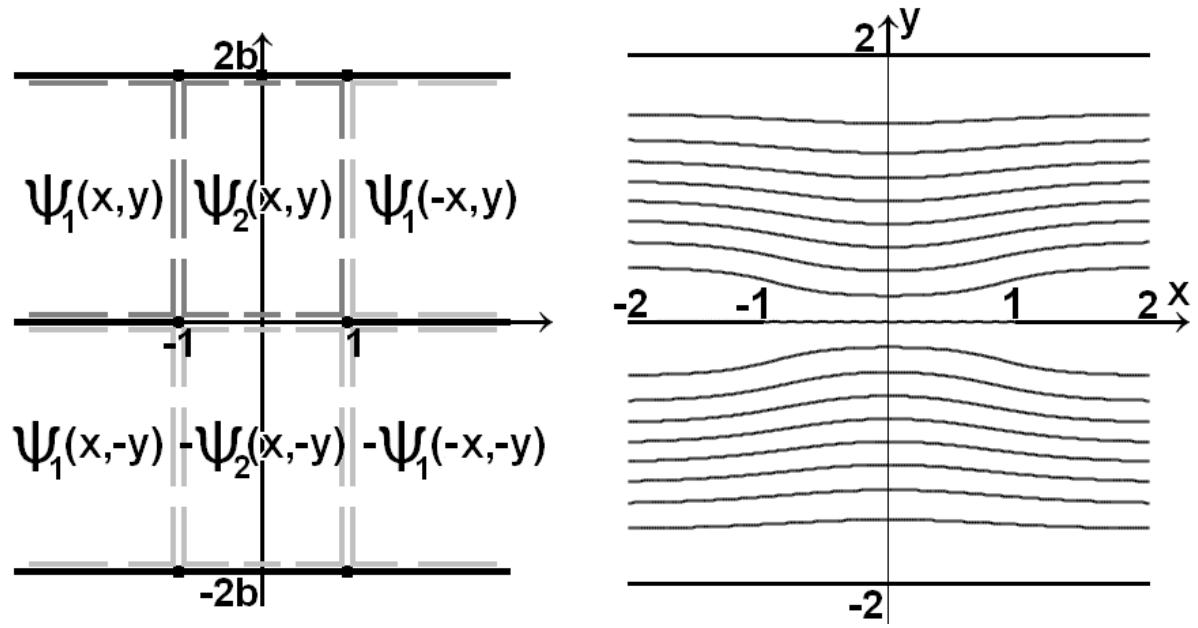

FiguRE 4. Left: diagram of the subregions we consider when constructing the flow profile in Figure 3(a). Right: plot of the streamfunction $\Psi$ associated with this flow profile when the shunt width equals the channel width and superimposed streamlines of the flow.

The terms $\tilde{f}(y)$ and $f\left(y_{1}\right)$ represent Poiseuille flow of maximum velocity 1 and mean velocity $2 / 3$ in a channel with boundaries at $y=0$ and $y=2 b$. We now construct the set of three linearly-independent flow solutions represented by the diagrams in Figure 3 . Due to the linearity of the biharmonic equations, a streamfunction $\Psi(x, y)$ can be constructed using appropriate infinite summations of terms that include Papkovich-Fadle eigenfunctions in both the $x$ and $y$ directions. We follow the notation and expansion forms that were used by Smith (1952) as well as the majority of authors mentioned in the introduction.

\subsection{First solution}

We first consider a solution to the flow represented by diagram (a) in Figure 3. The maximum velocity at each end is chosen to be 1 . This corresponds to the constants $c_{1}=4 b / 3, c_{2,3}=0$ and $c_{4}=-4 b / 3$ in Figure 2 . The boundary conditions that need to be satisfied are thus

$$
\begin{aligned}
\Psi=\frac{4 b}{3} \text { and } & \frac{\partial \Psi}{\partial y}=0 \quad \text { on } y=2 b \\
\Psi=0 & \text { and } \quad \frac{\partial \Psi}{\partial y}=0 \quad \text { on } y=0, x \leqslant-1 \text { and } y=0, x \geqslant 1 \\
\Psi=-\frac{4 b}{3} & \text { and } \quad \frac{\partial \Psi}{\partial y}=0 \quad \text { on } y=-2 b \\
\Psi & \rightarrow \tilde{f}(y) \quad \text { as }|x| \rightarrow \infty .
\end{aligned}
$$

The domain is divided into six subregions (Figure 4, left). As $\Psi$ is even in $x$ and odd in $y$, it is only necessary to derive the solution in two of the six regions,

$$
\Psi(x, y)=\left\{\begin{array}{ll}
\Psi_{1}(x, y) & \text { for } x \in(-\infty,-1], y \in[0,2 b] \\
\Psi_{2}(x, y) & \text { for } x \in[-1,1], y \in[0,2 b]
\end{array} .\right.
$$

Then we seek solutions using the following expansions

$$
\Psi_{1}\left(x, y_{1}\right)=f\left(y_{1}\right)+\sum_{n=1}^{\infty} \frac{A_{n}}{k_{n}^{2}} e^{k_{n}(x+1) / b} e_{1}^{n}\left(y_{1}\right)+\sum_{n=1}^{\infty} \frac{B_{n}}{K_{n}^{2}} e^{K_{n}(x+1) / b} e_{2}^{n}\left(y_{1}\right)
$$




$$
\begin{aligned}
\Psi_{2}\left(x, y_{1}\right)= & f\left(y_{1}\right)+\sum_{n=1}^{\infty} \frac{C_{n}}{k_{n}^{2}} \frac{\cosh \left(k_{n} x / b\right)}{\cosh \left(k_{n} / b\right)} e_{1}^{n}\left(y_{1}\right)+\sum_{n=1}^{\infty} \frac{D_{n}}{K_{n}^{2}} \frac{\cosh \left(K_{n} x / b\right)}{\cosh \left(K_{n} / b\right)} e_{2}^{n}\left(y_{1}\right) \\
& +\sum_{n=1}^{\infty} \frac{E_{n}}{b^{2} K_{n}^{2}} \frac{\cosh \left(K_{n} b y_{1}\right)}{\cosh \left(K_{n} b\right)} e_{2}^{n}(x)+\sum_{n=1}^{\infty} \frac{F_{n}}{b^{2} K_{n}^{2}} \frac{\sinh \left(K_{n} b y_{1}\right)}{\sinh \left(K_{n} b\right)} e_{2}^{n}(x) .
\end{aligned}
$$

It is assumed here and subsequently that complex conjugates are included in the series and all additional notation will be omitted. As the constants $k_{n}$ and $K_{n}$ are chosen to have positive real parts only, all terms in the expansions decay exponentially at the outlets except for the Poiseuille flow terms. We use eigenfunctions in both the $x$ and $y$-directions in the rectangular region $x \in[-1,1], y \in[0,2 b]$ with appropriate arguments for different channel widths. All terms in the expansions satisfy the biharmonic equation; therefore, if all boundary conditions and the matching between the subregions are satisfied then the problem is solved. Some boundary conditions are already satisfied by the choice of expansions. The remaining two are

$$
\Psi_{2}=\frac{4 b}{3} \quad \text { and } \quad \frac{\partial \Psi_{2}}{\partial y}=0 \quad \text { at } \quad y=2 b .
$$

At the interface $y=0$ the first and third derivatives are continuous by construction of the expansions. The other two continuity conditions need to be stipulated and they are equivalent to

$$
\Psi_{2}=0 \quad \text { and } \quad \frac{\partial^{2} \Psi_{2}}{\partial y^{2}}=0 \quad \text { at } \quad y=0 .
$$

At the interface $x=-1$ the continuity conditions up to the third derivative of $\Psi$ become

$$
\Psi_{1}=\Psi_{2}, \quad \frac{\partial \Psi_{1}}{\partial x}=\frac{\partial \Psi_{2}}{\partial x}, \quad \frac{\partial^{2} \Psi_{1}}{\partial x^{2}}=\frac{\partial^{2} \Psi_{2}}{\partial x^{2}} \quad \text { and } \quad \frac{\partial^{3} \Psi_{1}}{\partial x^{3}}=\frac{\partial^{3} \Psi_{2}}{\partial x^{3}} \quad \text { at } x=-1 .
$$

Substituting the expressions in equations (2.17-2.18) into equations (2.19-2.21) yield after some algebra eight equations for the unknown constants in the expansions. Four hold for all $x$ in the range $[-1,1]$ and four for all $y$ in the range $[0,2 b]$. The system can be transformed into the vector form

$$
\begin{aligned}
& \sum_{n=1}^{\infty}\left(E_{n}+F_{n}\right) \mathbf{e}_{2}^{n}(x)=-\sum_{n=1}^{\infty}\left(F_{n} \frac{\operatorname{coth}\left(K_{n} b\right)-K_{n}}{K_{n}}+E_{n} \frac{\tanh \left(K_{n} b\right)-K_{n}}{K_{n}}\right)\left[\begin{array}{c}
e_{2}^{n}(x) \\
0
\end{array}\right] \\
& \sum_{n=1}^{\infty} \frac{F_{n}-E_{n}}{2} \mathbf{e}_{2}^{n}(x)=\left[\begin{array}{c}
b \\
0
\end{array}\right]-\sum_{n=1}^{\infty} \frac{D_{n} \cos ^{2} K_{n}}{\cosh \left(K_{n} / b\right)}\left[\begin{array}{c}
\cosh \left(\frac{K_{n} x}{b}\right) \\
0
\end{array}\right]-\sum_{n=1}^{\infty} \frac{C_{n} \sin ^{2} K_{n}}{\cosh \left(k_{n} / b\right)}\left[\begin{array}{c}
\cosh \left(\frac{k_{n} x}{b}\right) \\
0
\end{array}\right](2 \\
& \sum_{n=1}^{\infty} \frac{C_{n}-A_{n}}{2} \mathbf{e}_{1}^{n}\left(y_{1}\right)+\sum_{n=1}^{\infty} \frac{D_{n}-B_{n}}{2} \mathbf{e}_{2}^{n}\left(y_{1}\right)=\sum_{n=1}^{\infty} \frac{E_{n} \cos ^{2} K_{n}}{\cosh \left(b K_{n}\right)}\left[\begin{array}{c}
\cosh \left(b K_{n} y_{1}\right) \\
0
\end{array}\right]+\sum_{n=1}^{\infty} \frac{F_{n} \cos ^{2} K_{n}}{\sinh \left(b K_{n}\right)}\left[\begin{array}{c}
\sinh \left(b K_{n} y_{1}\right) \\
0
\end{array}\right] \\
& \sum_{n=1}^{\infty} \frac{k_{n}\left(A_{n}+C_{n} \tanh \left(\frac{k_{n}}{b}\right)\right)}{2 b} \mathbf{e}_{1}^{n}\left(y_{1}\right)+\sum_{n=1}^{\infty} \frac{K_{n}\left(B_{n}+D_{n} \tanh \left(\frac{K_{n}}{b}\right)\right)}{2 b} \mathbf{e}_{2}^{n}\left(y_{1}\right)= \\
& =\sum_{n=1}^{\infty} \frac{E_{n} K_{n}^{2}}{\cosh \left(b K_{n}\right)}\left[\begin{array}{c}
\cosh \left(b K_{n} y_{1}\right) \\
0
\end{array}\right]+\sum_{n=1}^{\infty} \frac{F_{n} K_{n}^{2}}{\sinh \left(b K_{n}\right)}\left[\begin{array}{c}
\sinh \left(b K_{n} y_{1}\right) \\
0
\end{array}\right]
\end{aligned}
$$

Next we apply the biorthogonality properties by pre-multiplying both sides of the equations by the dyadic matrix and the adjoint vector of interest. Equations (2.22-2.23) for the even eigenfunction in the range $x \in(-1,1)$ yield two equations for every natural 
number $N$

$$
\begin{aligned}
& E_{N}+F_{N}=\sum_{n=1}^{\infty}\left(F_{n} \frac{\operatorname{coth}\left(K_{n} b\right)-K_{n}}{4 K_{n} \cos ^{4} K_{N}}+E_{n} \frac{\tanh \left(K_{n} b\right)-K_{n}}{4 K_{n} \cos ^{4} K_{N}}\right)\left\langle\mathbf{f}_{2}^{N}(x),\left[\begin{array}{c}
e_{2}^{n}(x) \\
0
\end{array}\right]\right\rangle \\
& E_{N}-F_{N}=\frac{2 b}{\cos ^{4} K_{N}}-\sum_{n=1}^{\infty} \frac{D_{n} \cos ^{2} K_{n}}{2 \cos ^{4} K_{N}}\left\langle\mathbf{f}_{2}^{N}(x),\left[\begin{array}{c}
\frac{\cosh \left(K_{n} x / b\right)}{\cosh \left(K_{n} / b\right)} \\
0
\end{array}\right]\right\rangle-\sum_{n=1}^{\infty} \frac{C_{n} \sin ^{2} k_{n}}{2 \cos ^{4} K_{N}}\left\langle\mathbf{f}_{2}^{N}(x),\left[\begin{array}{c}
\frac{\cosh \left(k_{n} x / b\right)}{\cosh \left(k_{n} / b\right)} \\
0
\end{array}\right]\right\rangle
\end{aligned}
$$

Integration of equations (2.24-2.25) between $y=0$ and $y=2 b$ with respect to the odd eigenfunction yields

$$
\begin{aligned}
& A_{N}-C_{N}=\sum_{n=1}^{\infty} \frac{F_{n} \cos ^{2} K_{n}}{2 \sin ^{4} k_{N}}\left\langle\mathbf{f}_{1}^{N}\left(y_{1}\right),\left[\begin{array}{c}
\frac{\sinh \left(b K_{n} y_{1}\right)}{\sinh \left(b K_{n}\right)} \\
0
\end{array}\right]\right\rangle \\
& A_{N}+C_{N} \tanh \left(\frac{k_{N}}{b}\right)=-\sum_{n=1}^{\infty} \frac{F_{n} b K_{n}^{2}}{2 k_{N} \sin ^{4} k_{N}}\left\langle\mathbf{f}_{1}^{N}\left(y_{1}\right),\left[\frac{\sinh \left(b K_{n} y_{1}\right)}{\sinh \left(K_{n} b\right)}-\right\rangle\right. \text {. }
\end{aligned}
$$

Therefore each constant $A_{N}$ or $C_{N}$ can be expressed as a sum of the constants $F_{N}$ and can be decoupled from any computations. Similar analysis of equations (2.24-2.25) but with respect to the even eigenfunction $e_{2}^{N}\left(y_{1}\right)$ gives

$$
\begin{aligned}
B_{N}-D_{N} & =\sum_{n=1}^{\infty} \frac{E_{n} \cos ^{2} K_{n}}{2 \cos ^{4} K_{N}}\left\langle\mathbf{f}_{2}^{N}\left(y_{1}\right),\left[\begin{array}{c}
\frac{\cosh \left(b K_{n} y_{1}\right)}{\cosh \left(b K_{n}\right)} \\
0
\end{array}\right]\right\rangle \\
B_{N}+D_{N} \tanh \left(\frac{K_{N}}{b}\right) & =-\sum_{n=1}^{\infty} \frac{E_{n} b K_{n}^{2}}{2 K_{N} \cos ^{4} k_{N}}\left\langle\mathbf{f}_{2}^{N}\left(y_{1}\right),\left[\begin{array}{c}
\frac{\cosh \left(b K_{n} y_{1}\right)}{\cosh \left(K_{n} b\right)} \\
0
\end{array}\right]\right\rangle .
\end{aligned}
$$

The constants $B_{N}$ and $D_{N}$ can be expressed in terms of the constants $E_{n}$. Thus the remaining equations $(2.26-2.27)$ can be written as an infinite set of linear equations in $E_{n}$ and $F_{n}$. An approximation of the solution is obtained by truncating the expansions to $N_{T}$ terms. The behaviour away from the sharp edges is captured by relatively few terms while accuracy at $( \pm 1,0)$ is limited by a Gibbs phenomenon. Such truncations are justified by many authors including Joseph (1977) and Joseph \& Sturges (1978). We find that the error of the terms $A_{1}, A_{2}$ and $B_{1}$ in the expansion as $N_{T}$ increases behaves as $N_{T}{ }^{-1.5}$. Results presented here are calculated with the truncation $N_{T}=40$. Figure 4 shows the streamfunction $\Psi(x, y)$ for $b=1$. By construction, the flow is symmetric about both the $x$ and $y$ axes, and is from left to right in both channels. The flow is not unidirectional, as would be the case if the shunt were not there. In the vicinity of the shunt the flow is affected by the change in boundary condition: it is the second derivative of $\Psi$ not the first that is zero along the shunt. The flow converges towards a Poiseuille profile on the length-scale of the shunt width as expected for low-Reynolds-number flows.

\subsection{Second solution}

The second solution we consider is characterised by Poiseuille flow in only one of the two channels. This breaks the symmetry in the flow about $y=0$. Without loss of generality, we assume that it is the bottom channel that would have had zero flow in it if the shunt were not present (diagram (b) in Figure 3).

The boundary conditions are shown in Figure 2 with $c_{1}=4 b / 3, c_{2}=c_{3}=c_{4}=0$. The flow is symmetric about $x=0$, so it is sufficient to consider four subregions only (Figure 
5 , left). The expansions are similar to the those in the previous section,

$$
\begin{aligned}
\Psi_{1}\left(x, y_{1}\right)= & f\left(y_{1}\right)+\sum_{n=1}^{\infty} \frac{A_{n}}{k_{n}^{2}} e_{1}^{n}\left(y_{1}\right) e^{k_{n}(x+1) / b}+\sum_{n=1}^{\infty} \frac{B_{n}}{K_{n}^{2}} e_{2}^{n}\left(y_{1}\right) e^{K_{n}(x+1) / b} \\
\Psi_{2}\left(x, y_{1}\right)= & f\left(y_{1}\right)+\sum_{n=1}^{\infty} \frac{E_{n}}{k_{n}^{2}} e_{1}^{n}\left(y_{1}\right) \frac{\cosh \left(k_{n} x / b\right)}{\cosh \left(k_{n} / b\right)}+\sum_{n=1}^{\infty} \frac{F_{n}}{K_{n}^{2}} e_{2}^{n}\left(y_{1}\right) \frac{\cosh \left(K_{n} x / b\right)}{\cosh \left(K_{n} / b\right)} \\
& +\sum_{n=1}^{\infty} \frac{G_{n}}{b^{2} K_{n}^{2}} \frac{\cosh \left(K_{n} b y_{1}\right)}{\cosh \left(K_{n} b\right)} e_{2}^{n}(x)+\sum_{n=1}^{\infty} \frac{H_{n}}{b^{2} K_{n}^{2}} \frac{\sinh \left(K_{n} b y_{1}\right)}{\sinh \left(K_{n} b\right)} e_{2}^{n}(x) \\
\Psi_{3}\left(x, y_{2}\right)= & \sum_{n=1}^{\infty} \frac{C_{n}}{k_{n}^{2}} e_{1}^{n}\left(y_{2}\right) e^{k_{n}(x+1) / b}+\sum_{n=1}^{\infty} \frac{D_{n}}{K_{n}^{2}} e_{2}^{n}\left(y_{2}\right) e^{K_{n}(x+1) / b} \\
\Psi_{4}\left(x, y_{2}\right)= & \sum_{n=1}^{\infty} \frac{I_{n}}{k_{n}^{2}} e_{1}^{n}\left(y_{2}\right) \frac{\cosh \left(k_{n} x / b\right)}{\cosh \left(k_{n} / b\right)}+\sum_{n=1}^{\infty} \frac{J_{n}}{K_{n}^{2}} e_{2}^{n}\left(y_{2}\right) \frac{\cosh \left(K_{n} x / b\right)}{\cosh \left(K_{n} / b\right)} \\
& +\sum_{n=1}^{\infty} \frac{L_{n}}{b^{2} K_{n}^{2}} \frac{\cosh \left(K_{n} b y_{2}\right)}{\cosh \left(K_{n} b\right)} e_{2}^{n}(x)+\sum_{n=1}^{\infty} \frac{M_{n}}{b^{2} K_{n}^{2}} \frac{\sinh \left(K_{n} b y_{2}\right)}{\sinh \left(K_{n} b\right)} e_{2}^{n}(x)
\end{aligned}
$$

where $y_{2}$ is defined in equation 2.11. There are sixteen equations that need to be satisfied by these expansions. By construction, $\Psi$ satisfies the boundary conditions on all rigid walls apart from $\{x \in[-1,1], y=2 b\}$ and $\{x \in[-1,1], y=2 b\}$. These provide two equations for $\Psi_{2}(x, y)$ and two for $\Psi_{4}(x, y)$. There are also three interfaces that require continuity conditions. As before, the solutions on both sides of such interfaces are matched up to the third derivative. This yields eight equations for the two interfaces at $x=-1$ and four equations at $y=0$. Integration is performed for each eigenfunction as before. All constants decouple apart from $G_{n}, H_{n}, L_{n}$ and $M_{n}$. These form a system of linear equations that can be solved after truncation to 32 terms in each summation.

Plots of $\Psi$ for $b=1, b=1 / 2$ and $b=1 / 3$ are shown in Figure 5. The contour lines in the top channel are uniformly spaced in the range $\left[0, \frac{4 b}{3}\right]$, whereas those in the lower channel are uniformly spaced in different, much lower in amplitude, ranges. The flow is dominated by the Poiseuille term, as expected. A relatively small amount of fluid crosses into the bottom channel: the streamline that joins the two shunt edges serves as the free boundary for the fluid in the two channels. There would be no flow in the bottom channel were it not for the shunt and the shear that the flow above exerts. The closed streamlines indicate that there exist weak circulatory regions in the bottom channel. They are the well-known Moffatt eddies that form in low-Reynolds-number flow in any corner of angle less than approximately 146 degrees (Moffatt 1963), where our solution corresponds to a "corner" of zero angle. Our analytic solution suggests that the eddies are of the same size for large $x$ and that the distance between them is determined by the imaginary part of the smallest root $K_{n}$ or $k_{n}$, i.e. $\Im\left\{K_{1}\right\} \approx 1.1254$. Moreover, the amplitude of the streamfunction inside the eddies decays exponentially as $\exp \left(-\Im\left\{K_{1}\right\}|x| / b\right)$ for large $|x|$.

\subsection{Third solution}

The third, and final, solution to be considered is represented by diagram (c) in Figure 3. Unlike the first two flows, it introduces flow of non-zero net flux through the shunt, which implies that it is linearly-independent of the previous solutions. In addition, it is symmetric about both the $x$ and $y$ axes. A solution can be obtained by considering two subregions of the geometry, as shown in Figure 6 . Note that this is the first decomposition where one region is of height $4 b$ rather than $2 b$ so that some eigenfunctions have argument $y_{3}=y / 2 b$. This permits the Poiseuille flow term to be expanded as a linear combination 


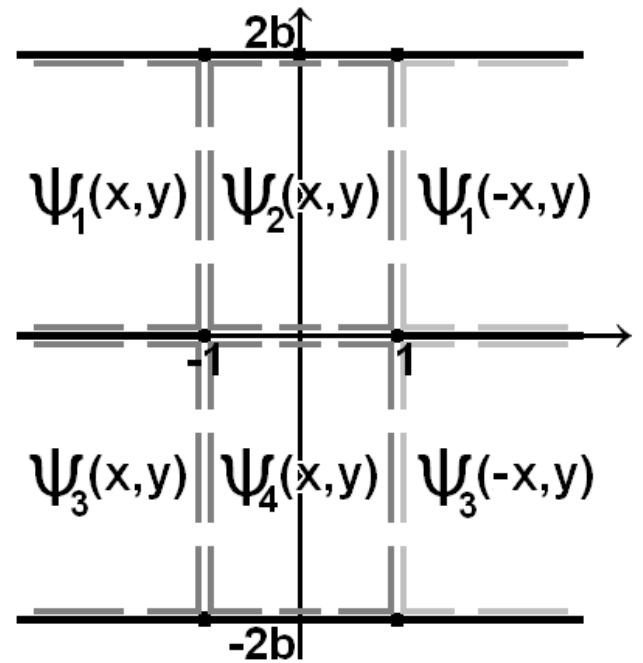

(a)

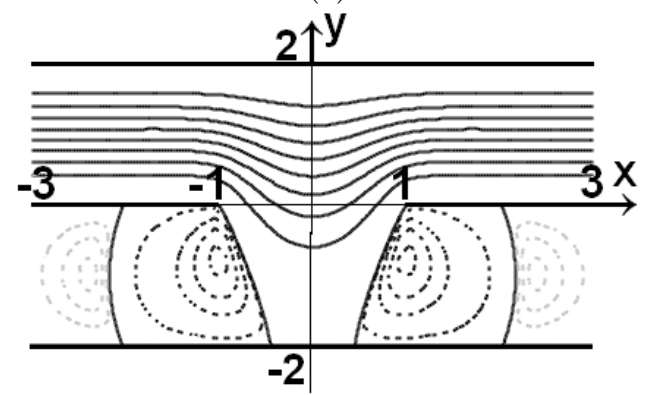

(c) $b=1 / 2$

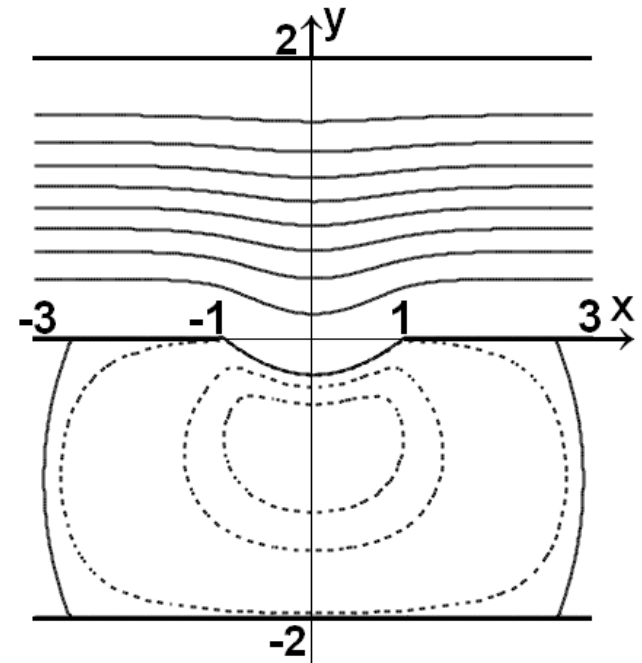

(b) $b=1$

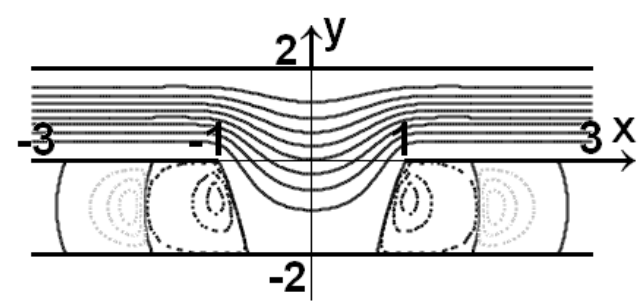

(d) $b=1 / 3$

Figure 5. (a) Diagram of the subregions we consider when constructing the flow profile in Figure 3(b). (b) Streamlines of the solution when $b=1$; The solid contours correspond to $\Psi=(4 / 3) \times(n / 9), n=0 . .9$ and the dotted contours to $\Psi=-2 \times 10^{-4}-0.06 \times(n / 4), n=0 . .4$. (c) Streamlines when $b=1 / 2$; The solid contours correspond to $\Psi=(4 / 3) \times(n / 9), n=0 . .9$, the dotted black contours to $\Psi=-6.4 \times 10^{-4}-6.4 \times 10^{-3} \times n, n=0 . .3$ and the dotted grey contours to $\Psi=1.6 \times 10^{-5}+2 \times 10^{-5} \times n, n=0 . .2$. (d) Streamlines when $b=1 / 2$; The solid contours correspond to $\Psi=(4 / 3) \times(n / 9), n=0 . .9$, the dotted black contours to $\Psi=-8 \times 10^{-5}-8 \times 10^{-3} \times n, n=0 . .2$ and the dotted grey contours to $\Psi=8 \times 10^{-6}+1.8 \times 10^{-5} \times n, n=0 . .2$.

of eigenfunctions. The boundary conditions associated with this third solution are shown in Figure 2 where the constants are $c_{1}=c_{4}=0, c_{3}=-4 b / 3$ and $c_{2}=4 b / 3$.

The solutions in the two subregions can be represented in the following way:

$$
\begin{aligned}
& \Psi_{1}\left(x, y_{1}\right)=f\left(y_{1}\right)-\frac{4 b}{3}+\sum_{n=1}^{\infty} \frac{A_{n}}{k_{n}^{2}} e_{1}^{n}\left(y_{1}\right) e^{k_{n}(1-x) / b}+\sum_{n=1}^{\infty} \frac{B_{n}}{K_{n}^{2}} e_{2}^{n}\left(y_{1}\right) e^{K_{n}(1-x) / b} \\
& \Psi_{2}\left(x, y_{3}\right)=\sum_{n=1}^{\infty} \frac{C_{n}}{K_{n}^{2}} e_{2}^{n}\left(y_{3}\right) \frac{\sinh \left(K_{n} x / 2 b\right)}{\sinh \left(K_{n} / 2 b\right)}
\end{aligned}
$$

Note that there is no explicit Poiseuille flow term in the second expansion and that the two expressions contain eigenfunctions that are biorthogonal over different regions, as indicated by the arguments $y_{1}$ and $y_{3}$. In addition, the chosen forms already consist of relatively few constants: three compared to six and twelve in the previous two sections. All no-slip conditions are satisfied by the choice of expansion. As before, we match at 

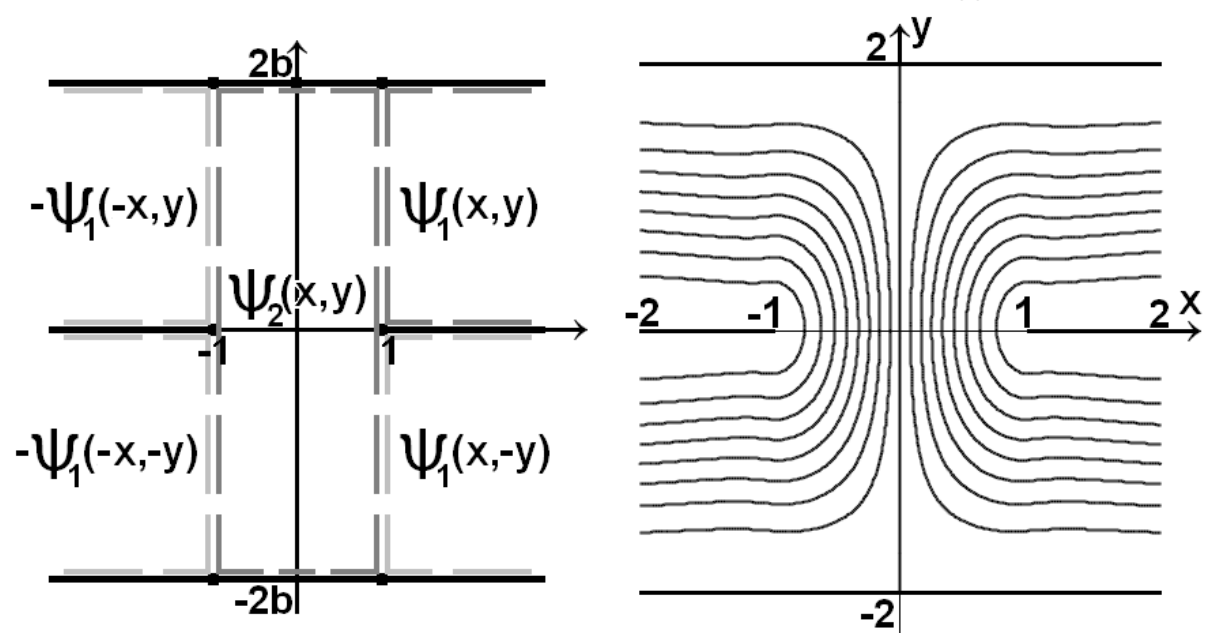

FiguRE 6. Left: diagram of the subregions we consider when constructing the flow profile in Figure 3(c). Right: plot of the streamfunction $\Psi$ associated with this flow profile when $b=1$.

the interface $x=1$ to ensure the continuity of the streamfunction and its first three $x$-derivatives. This is equivalent to

$$
\begin{aligned}
\sum_{n=1}^{\infty} \frac{C_{n}}{K_{n}^{2}} e_{2}^{n}\left(y_{3}\right) & =f\left(y_{1}\right)-\frac{4 b}{3}+\sum_{n=1}^{\infty} \frac{A_{n}}{k_{n}^{2}} e_{1}^{n}\left(y_{1}\right)+\sum_{n=1}^{\infty} \frac{B_{n}}{K_{n}^{2}} e_{2}^{n}\left(y_{1}\right)(23 \\
\sum_{n=1}^{\infty} \frac{C_{n} \operatorname{coth}\left(K_{n} / 2 b\right)}{2 K_{n}} e_{2}^{n}\left(y_{3}\right) & =-\sum_{n=1}^{\infty} \frac{A_{n}}{k_{n}} e_{1}^{n}\left(y_{1}\right)-\sum_{n=1}^{\infty} \frac{B_{n}}{K_{n}} e_{2}^{n}\left(y_{1}\right) \\
\sum_{n=1}^{\infty} \frac{C_{n}}{4} e_{2}^{n}\left(y_{3}\right) & =\sum_{n=1}^{\infty} A_{n} e_{1}^{n}\left(y_{1}\right)+\sum_{n=1}^{\infty} B_{n} e_{2}^{n}\left(y_{1}\right) \\
\sum_{n=1}^{\infty} \frac{C_{n} K_{n} \operatorname{coth}\left(K_{n} / 2 b\right)}{8} e_{2}^{n}\left(y_{3}\right) & =-\sum_{n=1}^{\infty} A_{n} k_{n} e_{1}^{n}\left(y_{1}\right)-\sum_{n=1}^{\infty} B_{n} K_{n} e_{2}^{n}\left(y_{1}\right)
\end{aligned}
$$

As before, the biorthogonality condition is used to obtain a system of linear equations. There is one difference: this time the first two and last two equations are coupled together before integration. As suggested by Phillips (1989), the two equations that match the second and third derivatives of the streamfunction should be analysed with respect to the bases $e_{1}^{n}\left(y_{1}\right)$ and $e_{2}^{n}\left(y_{1}\right)$. Both the $\Psi$ and its first derivative are continuous at the shunt edges $( \pm 1,0)$. Therefore integration can be performed with respect to the eigenfunction $e_{2}^{n}\left(y_{3}\right)$. Equations (2.38-2.41) are first rewritten in vector form after differentiation of $(2.38)$ and $(2.40)$

$$
\begin{gathered}
\sum_{n=1}^{\infty} \frac{C_{n}}{4} \mathbf{e}_{2}^{n}\left(y_{3}\right)=\left[\begin{array}{c}
0 \\
2 b y_{1}
\end{array}\right]+\sum_{n=1}^{\infty} \frac{C_{n}\left(K_{n}-2 \operatorname{coth}\left(\frac{K_{n}}{2 b}\right)\right)}{4 K_{n}}\left[\begin{array}{c}
e_{2}^{n}\left(y_{3}\right) \\
0
\end{array}\right]+\sum_{n=1}^{\infty} A_{n}\left[\begin{array}{c}
-e_{1}^{n}\left(y_{1}\right) / k_{n} \\
e_{1}^{n \prime \prime}\left(y_{1}\right) / k_{n}^{2}
\end{array}\right]+\sum_{n=1}^{\infty} B_{n}\left[\begin{array}{c}
-e_{2}^{n}\left(y_{1}\right) / K_{n} \\
e_{2}^{n \prime \prime}\left(y_{1}\right) / K_{n}^{2}
\end{array}\right] \\
\sum_{n=1}^{\infty} A_{n} k_{n}^{2} \mathbf{e}_{1}^{n}\left(y_{1}\right)+\sum_{n=1}^{\infty} B_{n} K_{n}^{2} \mathbf{e}_{2}^{n}\left(y_{1}\right)= \\
\sum_{n=1}^{\infty} A_{n} k_{n}\left(k_{n}-1\right)\left[\begin{array}{c}
e_{1}^{n}\left(y_{1}\right) \\
0
\end{array}\right]+\sum_{n=1}^{\infty} B_{n} K_{n}\left(K_{n}-1\right)\left[\begin{array}{c}
e_{2}^{n}\left(y_{1}\right) \\
0
\end{array}\right] \\
+\sum_{n=1}^{\infty} \frac{C_{n}}{16}\left[\begin{array}{c}
-2 K_{n} \operatorname{coth}\left(\frac{K_{n}}{2 b}\right) e_{2}^{n}\left(y_{3}\right) \\
e_{2}^{n \prime \prime}\left(y_{3}\right)
\end{array}\right] .
\end{gathered}
$$


Equation (2.42) is then premultiplied by the adjoint eigenvector corresponding to $\mathbf{e}_{2}^{n}(y / 2 b)$ and integration is performed over the range $y \in(0,2 b)$. In addition, equation (2.43) forms two equations after similar integration with respect to the eigenvectors $e_{1}^{n}\left(y_{1}\right)$ and $e_{2}^{n}\left(y_{1}\right)$ respectively. This yields three infinite sets of linear equations for $A_{n}, B_{n}$ and $C_{n}$. Figure 6 shows the streamlines of the flow when the summations are truncated to 20 terms. By construction, the solution is even in $y$ and odd in $x$.

This third solution is a special case $(\alpha=0)$ of the geometry considered by Cachile et al. (2012), where the four channels meet at an angle $\alpha$. Their numerical results suggest that for small enough $\alpha$ there will be at least three recirculation regions in the vicinity of the shunt. These disappear when $\alpha=0$, but they may be important when a side-to-side anastomosis connects non-parallel segments of the intestine.

Having found the solutions for three linearly independent cases, we can now derive the solution for any values of $c_{1}, c_{2}, c_{3}$ and $c_{4}$. First, we focus on the solutions that are relevant to modelling a side-to-side anastomosis.

\section{Application: a side-to-side anastomosis in the gut}

Side-to-side anastomoses are used to permit a portion of the flow to bypass intestinal loops which are diseased or highly resistive. For patients with Crohn's disease, for example, a high proportion of fluid is shunted, whereas other procedures require lesser amounts. The flow in the small intestine varies slowly in time with a Reynolds number of the order $10^{-3}$. Therefore the solutions that were derived in Sections 2.1-2.3 can be used as a basis to form quasi-steady solutions. The streamfunction of the full solution then defines the velocity, pressure and shear stress of the flow. This section is mainly interested in the flow properties as the fraction of flow through the shunt changes. This is biologically an interesting problem since the size of the surgical cut during a side-to-side anastomosis essentially determines the volume fraction for the particular fluid that passes through the top channel. We first consider results calculated for the particular case when the width of the shunt is the same size as the height of the channel, i.e. $L^{d}=1.25 \times 10^{-2} \mathrm{~m}$. This is realistic since usually the surgical cuts are of similar size to the diameter of the vessels.

The three constants of the solution are defined by the inlet and outlet fluxes in the system. One constant is prescribed to be such that the flux exiting the top right channel reenters through the bottom right channel to model the intestinal bend in Figure 1, i.e. $c_{1}=c_{4}$. The second constant is chosen so that the flow entering the system through the top left channel has maximum velocity 1 and non-dimensional flux $4 b / 3$. This leaves one free parameter and it determines the percentage of flow that bypasses the loop to the right of the shunt. The plots in Figure 7 show the streamlines for varying shunt fluxes: the first plot is for the case when $40 \%$ of the flow bypasses the intestinal loop and the last when the entire flow is diverted through the shunt. As the Poiseuille flow is of varying size in each case, the pressure gradient tends to different constants at the inlets and outlets.

The non-dimensional pressure along the middle of the top channel for varying shunt flows is shown in Figure 8, where the pressure scale is $\mu^{d} \tilde{U}^{d} / \tilde{L}^{d} / 2$. The pressure curves are separated by an arbitrary integration constant. The pressure gradients are identical and tend to -2 as $x^{d}$ approaches the inlet to the left. Downstream the pressure gradients differ according to the bypass flow percentage, varying from -2 to 0 for $0 \%$ to $100 \%$.

At the shunt edges, the streamfunction and velocity in Stokes flow are finite, but higher derivatives are singular. We denote the edge by $\theta= \pm \pi$ in terms of local polar coordinates $(r, \theta)$ about the sharp edge. Then to leading order as $r \rightarrow 0, \psi \simeq A r^{3 / 2} \cos ^{2}(\theta / 2) \sin [(\theta-$ $\alpha) / 2$, where the constants $A$ and $\alpha$ are determined globally. Thus a streamline can leave 


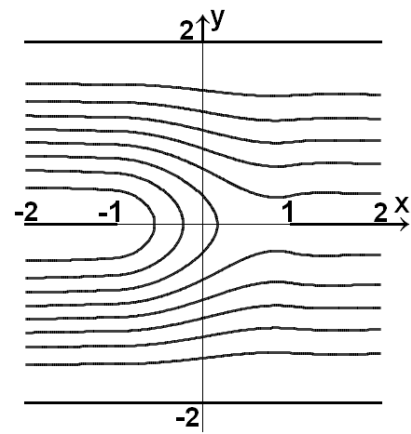

(a)

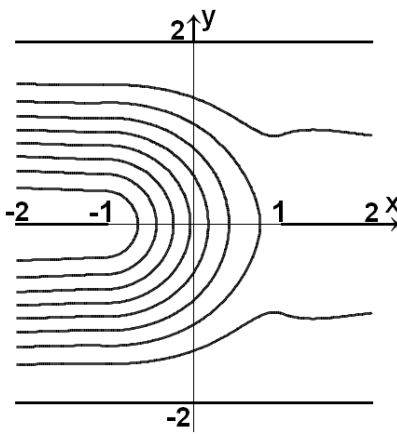

(b)

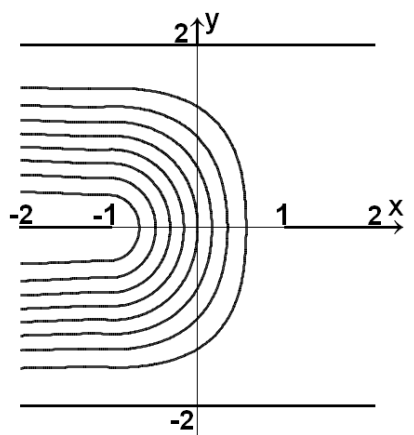

(c)

FIGURE 7. Streamlines of flow in a side-to-side anastomosis for different flux ratios of flow bypassing the intestinal loop over the net flow entering the domain through the top left extremity: $40 \%$ (a), $80 \%$ (b) and $100 \%$ (c).

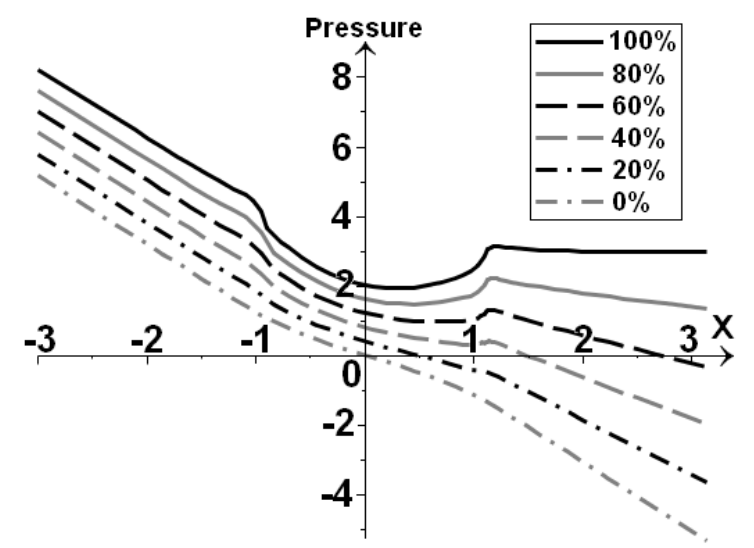

Figure 8. Pressure along the middle of the top channel when $b=1$ for different flux ratios of flow through the shunt.

the edge at any angle $\theta=\alpha$, while the pressure, vorticity and shear stress have a squareroot singularity. The amplitude $A$ varies exactly linearly with the flux through the shunt. It increases for the edge at $(x=-1)$ and decreases for the edge at $x=1$ as suggested by figure 7 . However, our spectral truncation is worst at the singularities, and it is hard to quantify the singularity very accurately.

The analytical model can be used to compare results for different shunt widths. We present results for the change in flow profile with respect to this width when the flux through the shunt is zero (first column in Figure 9) and when the flux through the intestinal bend equals that escaping through the anastomosis (second column in Figure 9. The top of the second column $(b=1)$ illustrates a typical desired outcome, where almost all the flow passes either through the shunt or round the loop. As the width $b$ increases, however, a substantial closed circulatory region develops in the loop as shown in the lower two plots in the second column. Such regions are potentially damaging to the tissue either by increasing the residence time of toxins or by facilitating the development of infection in the loop. Despite the increased health risk, there is a temptation to use such large cuts when the objective is accelerated weight-loss.

The first column of results reveals a similar effect even when there is no flux through the gap. As the shunt width increases, the zero net flux is maintained by two counter- 

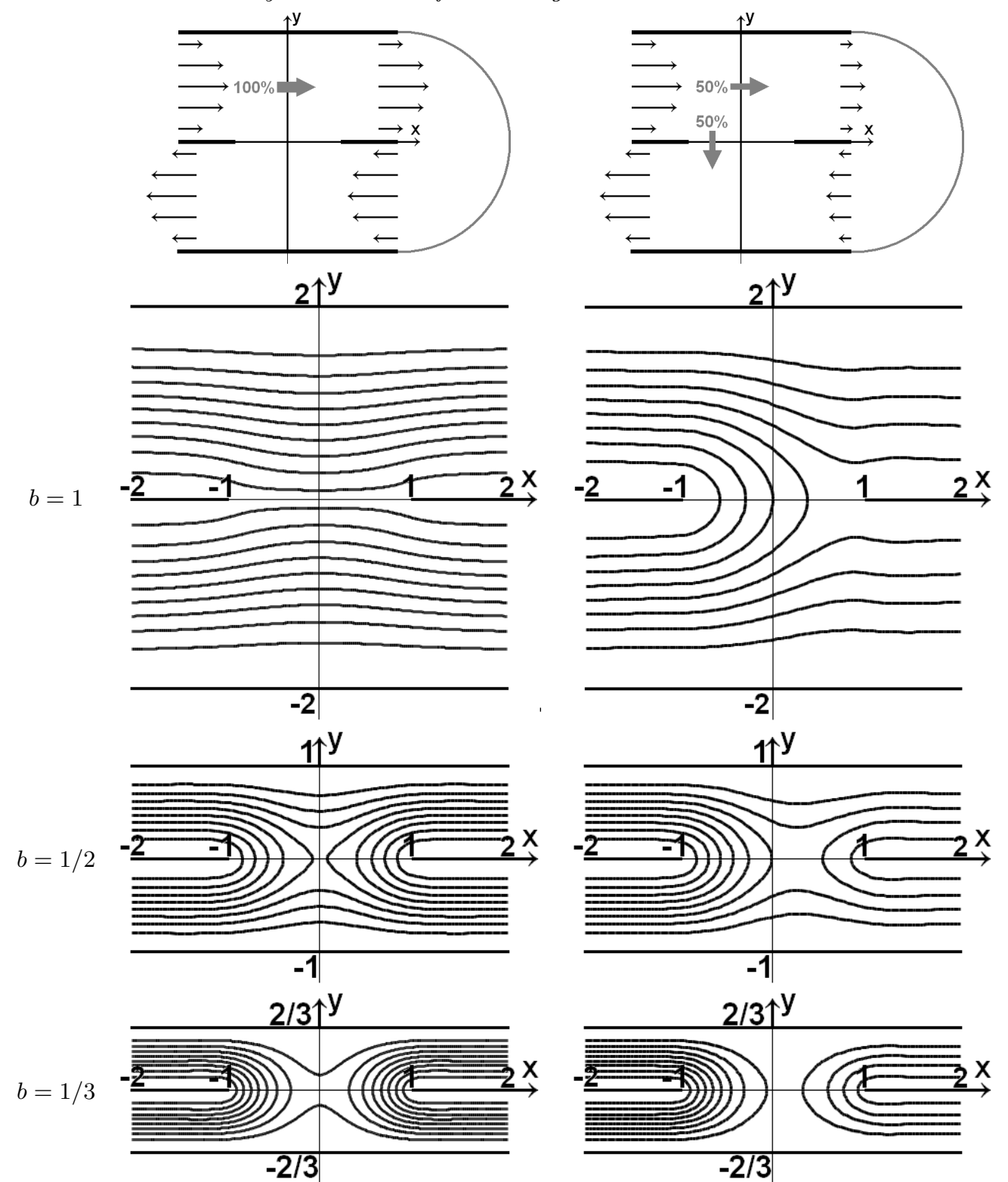

FiguRE 9. Top row: Schematic drawings of flow round an intestinal bend when there is no net flux through the shunt (left) and when $50 \%$ of the total flux entering the intestine through the top left channel is bypassed through the shunt (right). The plots below the drawings correspond to streamlines of the flows with such flow distribution when $b=1$ (second row), $b=1 / 2$ (third row) and $b=1 / 3$ (fourth row).

rotating disconnected loops of increasing magnitude. The flow profiles in this first column can be obtained by superimposing the solution found in Section 2.2 and its inverted image in the opposite direction. When the shunt is large the Poiseuille profile entering the top channel has sufficient distance to develop into Poiseuille flow spanning the wider channel. Then the inverted profile added there results in cancellation of flow along and near the line $x=0$, and thus also in the aforementioned recirculating regions to the right of 
the anastomoses. If there are multiple such large shunts, then we expect that multiple recirculating regions will also occur. This concurs with many clinical observations that multiple smaller anastomoses are more successful than a single larger one.

\section{Conclusion}

Flow in the gut is not very well understood due to difficulties in measurements. Although abdominal surgical procedures are becoming more and more widespread, there is a wide range of surgical procedures and often subjective preference, experience and available resources dictate the choice of surgery rather than more scientific criteria. The model derived in this paper is intended as a first step toward the understanding and predicting the characteristics of one particular procedure, a side-to-side anastomosis. The analytical form of the solution allows us to investigate different parameters and regimes. It provides estimates for the velocity and pressure distributions in the small intestine based on an idealised two-dimensional geometry of a shunt, and therefore has the potential to be applied in the future to optimise key factors in surgery such as the position, size and resistance of shunts. Such models are important for the future success of invasive treatments.

Our emphasis on the side-to-side anastomosis means that we have not exhausted all linear combination of the three solutions derived in this paper. The model can be applied to other biological problems where low-Reynolds-number flow is shunted or leaks through a gap. For example, a similar two-dimensional geometry was considered by Adamson \& Michel (1993) for the flow through a discontinuity in the tight junction of an intercellular cleft. In addition, the mathematical model can be easily modified by imposing the closure of some of the channel ends to simulate any problem where low-Reynolds-number flow in one main channel leaks through a gap on one of the walls or into a single vessel. Such examples include Schlemm's canal in the eye and mixing channels in microfluidics. Other combinations of the three solutions in the model can be used to model the micro-vascular networks in the brain and, in particular, many of the redundant loops. Figure 2 shows three possible linear combinations when there is no flow in our out of some of the channel extremities, which can be used to model low-Reynolds-number flows with a blocked vessel a long way away from the shunt. The solutions (a), (b) and (c) correspond to the set of values $\{4 / 3,0,2 / 3,2 / 3\},\{4 / 3,0,2 / 3,0\}$ and $\{4 / 3,0,4 / 3,0\}$ for $\left\{c_{1}, c_{2}, c_{3}, c_{4}\right\}$ in Figure 2 respectively.

In this paper we have considered low-Reynolds-number flow in a two-dimensional geometry of two channels joined by a finite-sized shunt. Although for a particular geometry the problem is readily solved numerically, the analytic approach provides a solution for the entire range of $b$-values. The difficulty in studying this problem analytically is that a general solution of the biharmonic equation cannot be expressed using a single orthogonal basis of eigenfunctions. Progress is made by decomposing the domain into rectangular regions and considering Papkovich-Fadle expansions in each one. This method is usually used for geometries with only one inlet and one outlet and therefore the solutions in this paper are a natural extension to previous work. The methodology required to obtain the third solution in this paper is of particular interest since it uses two different bases in each rectangular region.

The authors would like to thank Professor Tim Phillips from Cardiff University for his invaluable help and many discussions on the theory of this paper. 


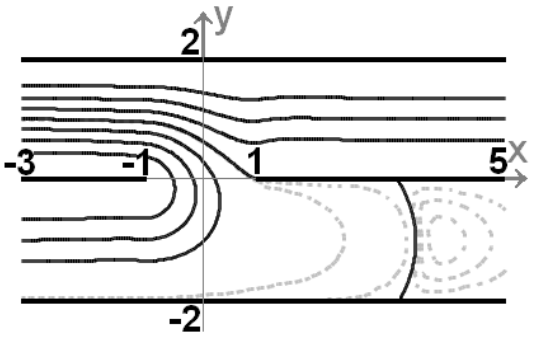

(a)

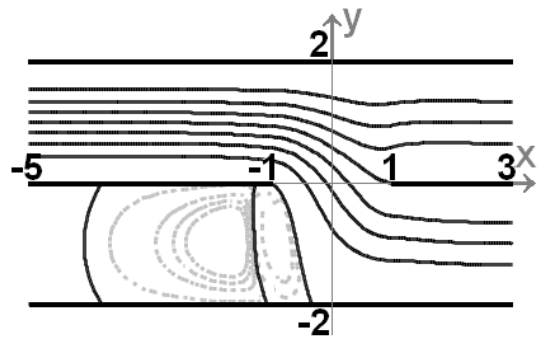

(b)

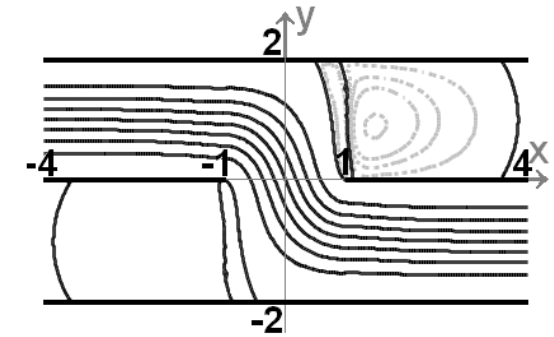

(c)

FiguRE 10. Flow streamlines when $b=1$ if one channel end is blocked ((a) and (b)) and if two diagonal channel ends are closed (c). The solid contours correspond to $\Psi=(4 / 3) \times(n / 8), n=0 . .8$ in all three plots. (a) The dotted grey contours correspond to $\Psi=2 / 3-5 \times 10^{-3}, 2 / 3-10^{-4}$ and $\Psi=2 / 3+10^{-5}, 2 / 3+3 \times 10^{-5}, 2 / 3+6 \times 10^{-5}$. (b) The dotted grey contours correspond to $\Psi=-5 \times 10^{-3},-10^{-3}$ and $\Psi=10^{-4}, 10^{-3}, 1.9 \times 10^{-3}, 2.8 \times 10^{-3}$. (c) The dotted grey contours correspond to $\Psi=4 / 3+2 \times 10^{-3}$ and $\Psi=4 / 3-10^{-3}, 4 / 3-5 \times 10^{-3}, 4 / 3-10^{-2}, 4 / 3-2 \times 10^{-2}$.

\section{REFERENCES}

Abrahams, I. D., Davis, A. M. J. \& Smith, S. G. Llewellyn 2008 Asymmetric channel divider in stokes flow. SIAM J. Appl. Math. 68 (5), 1439-1463.

Adamson, R. H. \& Michel, C. C. 1993 Pathways through the intercellular clefts of frog mesenteric capillaries. J. Physiol. 466, 303-327.

Buchwald, V. T. \& Doran, H. E. 1965 Eigenfunctions of plane elastostatics. ii. a mixed boundary value problem of the strip. Proc. Roy. Soc. Ser. A 284, 69-82.

Cachile, M., Talon, L., Gomba, J. M., Hulin, J. P. \& Auradou, H. 2012 Stokes flow paths separation and recirculation cells in x-junctions of varying angle. Phys. Fluids 24 (021704), $1-7$.

Crowdy, D. \& Samson, O. 2010 Stokes flows past gaps in a wall. Proc. R. Soc. A 466 (2121), $2727-2746$.

Davis, A. M. J. 1991 Shear flow disturbance due to a hole in the plane. Phys. Fluids. 3 (3), $478-480$.

Driesen, C. H., Kuerten, J. G. M. \& Streng, M. 1998 Low-reynolds-number flow over partially covered cavities. J. Eng. Math. 34 (1-2), 3-20.

Foote, R. M. L. \& Buchwald, V. T. 1985 An exact solution for the stress intensity factor for a double cantilever beam. Int. J. Fracture 29, 125-134.

JosePh, D. D. 1977 The convergence of biorthogonal series for biharmonic and stokes flow edge problems. part i. SIAM J. Appl. Math. 33 (2), 337-347.

Joseph, D. D. \& Sturges, L. 1975 The free surface on a liquid filling a trench heated from its side. J. Fluid Mech. 69, 565-590.

Joseph, D. D. \& Sturges, L. 1978 The convergence of biorthogonal series for biharmonic and stokes flow edge problems. part ii. SIAM J. Appl. Math. 34 (1), 7-26.

Joseph, D. D., Sturges, L. D. \& Warner, W. H. 1982 Convergence of biorthogonal series of biharmonic eigenfunctions by the method of titchmarsh. Arch. Rat. Mech. Anal. 78, 223-279. 
Joyce, M., Sweeney, K. J., Johnston, S. \& Geraghty, J. G. 2002 Techniques of bowel resection and anastomosis. CME J. Gynecol. Oncol. 7, 284-289.

KIM, S. \& KARRILA, S. J. 2005 Microhydrodynamics: principles and selected applications, 2nd edn. Dover Publications.

Kiran, R. P., Nisar, P. J., Church, J. M. \& Fazio, V. W. 2011 The role of primary surgical procedure in maintaining intestinal continuity for patients with crohn's colitis. Ann. Surg. 253 (6), 1130-1135.

Ko, H.-J. \& JEONG, J.-T. 1994 Two-dimensional slow stagnation flow near a slit. J. Phys. Soc. Jpn. 63 (9), 3288-3294.

Moffatt, H. K. 1963 Viscous and resistive eddies near a sharp corner. J. Fluid Mech. 18 (1), $1-18$.

Phillips, T. N. 1989 Singular matched eigenfunction expansions for stokes flow around a corner. IMA J. Appl. Math. 42 (1), 13-26.

PozRIKIDIS, C. 1992 Boundary integral and singularity methods for linearized viscous flow, 1st edn. Cambridge University Press.

Resegotti, A., Astegiano, M., Farina, E. C., Ciccone, G., Avagnina, G., Giustetto, A., Campra, D. \& Fronda, G. R. 2005 Side-to-side stapled anastomosis strongly reduces anastomotic leak rates in crohn's disease surgery. Dis. Colon Rectum. 48 (3), 464-468.

Setchi, A. T., Mestel, A. J., Siggers, J. H., Parker, K. H., Tan, M. W. \& Wang, K. 2012 Mathematical model of flow through the patent ductus arteriosus. Submitted to J. Math. Biol. in March 2012.

Sмith, R. C. T. 1952 The bending of a semi-infinite strip. Aust. J. Sci. Res. 5, 227-237.

Smith, S. H. 1987 Stokes flow past slits and holes. Int. J. Multiphase Flow 13, 219-231.

Souba, W. W., Fink, M. P., Jurkovic, G. J., Pearce, W. H., PemberTON, J. H. \& Soper, N. J., ed. 2007 ACS Surgery: Principles and Practice, 6th edn. Decker Publishing, particular contribution by N. J. Mortensen and S. Ashraf in Chapter 5.29 Intestinal Anastomosis. Image last accessed via http://206.47.151.137/bcdecker/figures/acs/thumb/part05_ch29_fig5.gif on 23/07/2012.

TrIPATHI, D. 2011 A mathematical model for the peristaltic flow of chyme movement in small intestine. Math. Biosci. 233 (2), 90-97.

Trogdon, S. A. \& Joseph, D. D. 1982 Matched eigenfunction expansions for a slow flow over a slot. J. Non-Newtonian Fluid Mech. 10, 185-213. 